\title{
Aortic Dissection Complicated with Fatal Cerebral Infarction: Case Report and Review of Literatures*
}

\author{
Kentaro Hayashi, Nobutaka Horie, Kazuhiko Suyama, Izumi Nagata \\ Department of Neurosurgery, School of Medicine, Nagasaki University, Nagasaki, Japan \\ Email: kenkuni@net.nagasaki-u.ac.jp
}

Received February 6, 2012; revised February 26, 2012; accepted March 2, 2012

\begin{abstract}
Acute aortic dissection is a life-threatening condition requiring immediate assessment and therapy. Rarely, aortic dissection involves carotid arteries and manifest cerebral infarction. Here, we report a case of aortic dissection complicated with fatal cerebral infarction. A 83-year-old man, who suddenly suffered consciousness disturbance and right hemiparesis, was transferred to our hospital for the treatment of stroke. Magnetic resonance image revealed massive cerebral infarction in the left cerebral hemisphere as well as occlusion of the left internal carotid artery. Duplex ultrasonography demonstrated arterial dissection in the bilateral carotid arteries and the blood flow was compromised especially in the left side. Aortic dissection was confirmed by the contrast enhanced computed tomography. He was treated conservatively and died of cerebral hernia three days after the onset. In conclusion, aortic dissection may involve carotid artery and results in cerebral infarction. Ultrasound screening can aid timely diagnosis of aortic dissection and further management.
\end{abstract}

Keywords: Aortic Dissection; Cerebral Infarction; Thrombolytic Therapy; Carotid Artery Occlusion

\section{Introduction}

Acute aortic dissection is a life-threatening condition requiring immediate assessment and therapy. A patient suffering from aortic dissection often present with an insignificant or irrelevant medical history, giving rise to possible misdiagnosis. Cerebral ischemic stroke is the most common neurologic manifestation associated with aortic dissection, affecting $10 \%-30 \%$ of patients [1,2]. Once the patient is diagnosed as having ischemic stroke, thrombolytic or anticoagulant therapy may be started before the true cause of stoke is determined. We treated a case of aortic dissection, who suffered hemiparesis and was transferred for the treatment of stroke. Carotid artery dissection was revealed by the carotid duplex ultrasonography. And CT angiography showed aortic dissection extended to the bilateral common carotid arteries (CCAs). We report the case and the reports on cerebral ischemia due to the aortic dissection were reviewed. Thus, the diagnostic tool and thrombolysis were discussed.

\section{Case Report}

A 83-year-old man, who had a history of hypertension, was brought to a local clinic suffering from a decreased level of consciousness. The patient had suddenly com-

\footnotetext{
*The authors have no personal financial or institutional interest in any of the drugs, materials or devices described in the article.
}

plained of general weakness eventually collapsing onto the ground. From that moment, the patient was unresponsive. Neurological examination revealed right hemiparesis. Brain computed tomography (CT) showed no hemorrhagic findings. Because of the clinical picture suggestive of stroke and a time interval of less than 3 hours from the onset of symptoms, the patient was considered for thrombolytic therapy. Then, he was transferred to our hospital.

On examination, the patient was in stupor (Glasgow Coma Scale 6), reacting only to pain. His blood pressure was 90/60 mmHg, pulse rate was 51 beats per minutes. Physical examination revealed right hemiparesis. There was no nuchal rigidity. The National Institute of Health Stroke Scale (NIHSS) score was 19 at that point. Brain magnetic resonance (MR) image was arranged immediately, which disclosed acute cerebral infarction in the left cerebral hemisphere (Figure 1(a)). Thus, the left internal carotid artery was not visualized in MR angiography (Figure 1(b)). Since hypotension and bradycardia were noted, the cause of the left internal carotid artery occlusion was examined carefully. The chest roentogram showed obvious widened mediatinum. The electrocardiogram did not show ischemic change. In the blood test, cardiac enzymes were within normal limits, while Ddimer was elevated as $109 \mathrm{ug} / \mathrm{ml}$. The duplex ultrasonography demonstrated intimal flap of the right CCA due to 


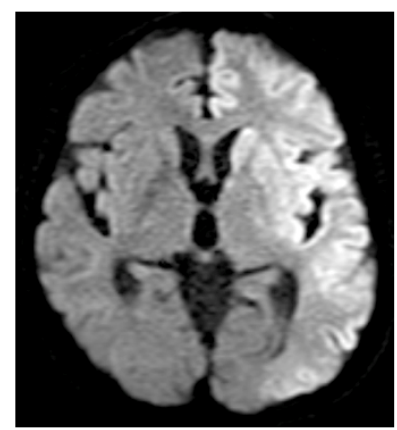

(a)

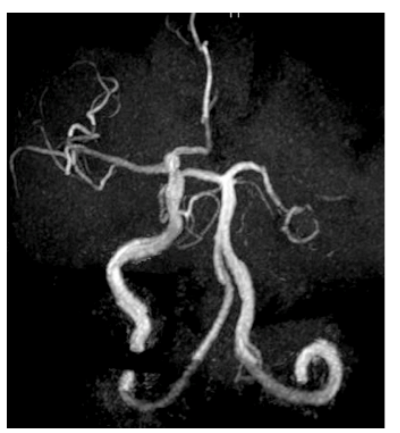

(b)
Figure 1. MR images. (a) Diffusion weighted image showed acute cerebral infarction in the left cerebral hemisphere; (b) MR angiography showed occlusion of the left internal carotid artery.

the arterial dissection and no flow signal in the left CCA (Figure 2). A CT scan with intravenous contrast revealed a Stanford type A dissection of the thoracic aorta with a propagation and significant occlusion of the left CCA (Figure 3). Because of the severe cerebral infarction and poor general condition, replacement of the aortic arch was not indicated. He was treated conservatively and died of cerebral hernia 3 days after the onset.

\section{Discussion}

Classically, acute aortic dissection usually presents with an abrupt onset of severe pain in the chest, back, or abdomen. Typically, the pain moves as the dissection gets worse. Nevertheless, aortic dissection is painless in about $10 \%$ of patients especially those with neurologic complications as presented by our patient [1-3].

Aortic dissection may extend into major branches and manifest symptom of the involved organs. Neurologic deficits have been associated with 10\% - 30\% cases of aortic dissection and are commonly attributable to ischemic cerebral infarcts, spinal cord ischemia, ischemic neuropathy and hypoxic encephalopathy [1]. Cerebral ischemia is the most common neurologic manifestation associated with aortic dissection and has been reported affect $10 \%-20 \%$ of patients [1]. Asouhidou et al. reported that among 49 patients with aortic dissection, three patients later found to be suffering an aortic dissection were initially diagnosed with cerebral infarction [4]. Right CCA occlusion is most frequently seen in approximately $80 \%$ of stroke cases [5]. In addition, Stanley et al. reported a case of severe aortic dissection that extended into the intracranial internal carotid artery [3]. Left CCA was severely involved in our patient and resulted massive infarction of the left cerebral hemisphere. Thus, our patient demonstrated hypotension and bradycardia. Usually, in case of ischemic stroke, hypertension is induced as systemic reaction. Cardiac failure, cardiac tamponade, and

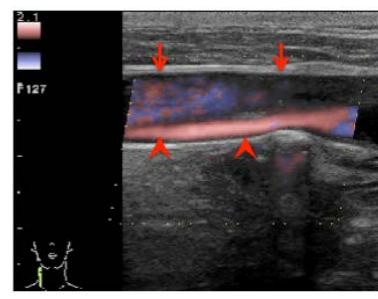

(a)

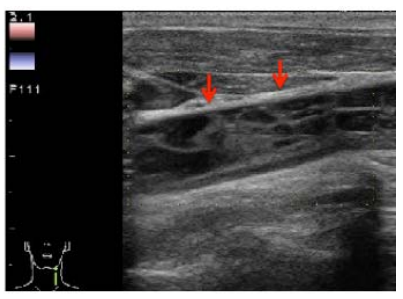

(b)
Figure 2. Sagittal images of duplex ultrasonography of the common carotid arteries. (a) Ultrasonography of the right common carotid artery demonstrated slow flow signal of false lumen (arrow) and normal flow signal of true lumen (arrow head). (b) Ultrasonography of the left common carotid artery demonstrated no flow signal (arrow).

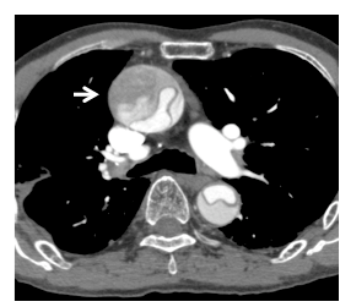

(a)

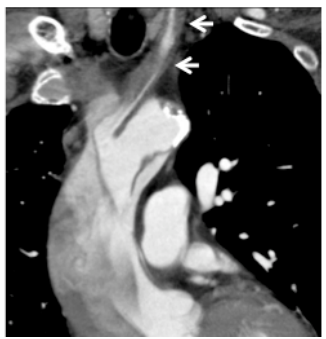

(c)

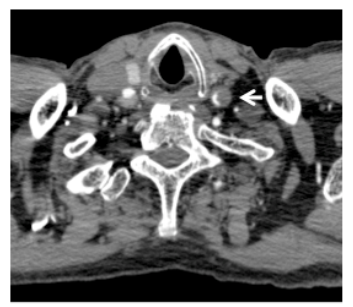

(b)

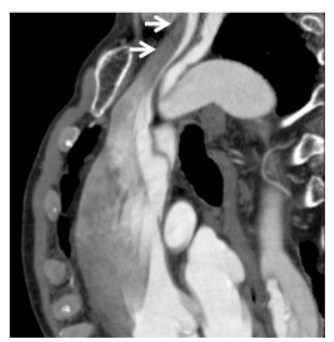

(d)
Figure 3. Post contrast CT images. (a) Axial image of the thorax revealed markedly dilated ascending aorta with unopacified false lumen (arrow). (b), (c), (d): Axial (b), coronal (c), sagittal (d) images of chest to neck demonstrated left common carotid arty with very narrow opacified true lumen (arrow).

rupture of aorta may be induced by aortic dissection and results in hypotension. Thus, carotid sinus may be affected by carotid dissection and hypotension or bradycardia may occur. Therefore, it is considered that hypotension is an important sign, which indicates ischemic stroke associated with aortic dissection.

There have been a number of cases of suspected myocardial infarction inadvertently treated with thrombolysis, complicated by extension of dissection into the pericardium, leading cardiac tamponade and death. The guidelines for thrombolysis in myocardial infarction mention aortic dissection as an absolute contraindication [6]. Recently, patients presenting with acute ischemic stroke within $3 \mathrm{~h}$ of symptom onset are considered for systemic thrombolysis. Same as heart disease, aortic rupture with 
Table 1. Table Key imaging for the diagnosis of aortic dissection and the findings in patient with acute cerebral ischemia.

\begin{tabular}{|c|c|c|}
\hline Reports & Key imaging & Findings \\
\hline Flemming $[10]$ & chest x-ray & widened superior mediastinum \\
\hline Kwon [13] & chest x-ray & post-mediastinal mass \\
\hline Kawarabuki [2] & carotid duplex ultrasonography & subintimal dissection with severe stenosis due to a false lumen of the CCA \\
\hline Iguchi [12] & carotid duplex ultrasonography & intimal flap of the CCA \\
\hline Rodríguez-Luna [14] & carotid duplex ultrasonography & double lumen of the proximal CCA \\
\hline present & carotid duplex ultrasonography & intimal flap and false lumen of the CCA \\
\hline Zaidat [15] & transesopahgeal echocardiography & aortic dissection with aortic valve insufficiency and tamponade \\
\hline Grupper [7] & chest CT with contrast & type A dissection of thoracic aorta \\
\hline Yeh $[11]$ & chest CT angiography & aortic dissection involving the CCA \\
\hline Wright [16] & chest CT & type A aortic dissection \\
\hline Stanley [3] & brain CT angiography & ICA dissection \\
\hline
\end{tabular}

CCA: common carotid artery, ICA: internal carotid artery.

dissection is the principal mechanism for early death, the use of thrombolysis for ischemic stroke is clearly contraindicated [1,7-9].

Thrombolysis must be administered within a very brief time window; therefore, it is important that patients with stroke mimics or major contraindications are identified quickly. The reports, which described the key imaging study for the diagnosis of aortic dissection, were reviewed and listed in Table 1. X-ray is a relatively simple and quick technique for establishing diagnosis, therefore, Flemming et al mentioned chest x-ray should be considered as part of ischemic stroke protocol [10]. However, up to $20 \%$ of chest X-ray may be negative in patients with aortic dissection [10]. Conventional, CT or MR angiography and transesophageal echocardiography are useful in the diagnosis of aortic dissection. We employed CT angiography for making definite diagnosis of the aortic dissection and dissection extending to the bilateral CCA was clearly demonstrated. However, some of them are time-consuming or not applicable for emergency treatment for stroke.

Ultrasonography is widely used in the medical treatment and is usually at hand in the Emergency Room [11]. It is a noninvasive, bedside, real-time diagnostic tool that also may help guide appropriate therapeutic decisions and avoid complications related to treatment. Established findings of arterial dissection of duplex ultrasonography are intramural hematoma, intimal flap, false lumen, and intraarterial floating signs in the CCA. Iguchi et al. reviewed cases of cerebral ischemia due to the aortic dissection and reported all 6 cases who underwent duplex ultrasonography had positive findings in CCA [12]. As shown in Figure 3, duplex ultrasonography revealed a subintimal dissection with severe stenosis due to a false lumen of the left carotid artery. After this experience, we always check carotid lesion using ultrasonography before ad- ministration of thrombolytic agent.

Stroke at presentation is not considered a contraindication for surgical intervention for aortic dissection, irespective of the extension of the dissecting process into the braches [5]. However, an evolving massive cerebral infarction has not been clearly addressed in this setting.

\section{Conclusion}

Aortic dissection should be carefully considered in ischemic stroke patients presenting with hypotension. Carotid ultrasonography is recommended as first-line imaging study for patient with cerebral infarction to differentiate aortic dissection.

\section{REFERENCES}

[1] C. Gaul, W. Dietrich, I. Friedrich, J. Sirch and F. J. Erbguth, "Neurological Symptoms in Type A Aortic Dissections,” Stroke, Vol. 38, No. 2, 2007, pp. 292-297. doi:10.1161/01.STR.0000254594.33408.b1

[2] K. Kawarabuki, T. Sakakibara, M. Hirai, M. Shirasu, I. Kohara, H. Tanaka, M. Oyamada, T. Takamatsu, Y. Murayama and T. Yamaki, "Acute Aortic Dissection Presenting as a Neurologic Disorder," Journal of Stroke and Cerebrovascular Diseases, Vol. 15, No. 1, 2006, pp. 26-29. doi:10.1016/j.jstrokecerebrovasdis.2005.09.001

[3] I. Stanley, V. K. Sharma, G. Tsivgoulis, A. Y. Lao and A. V. Alexandrov, "Painless Aortic Dissection with Unusual Extension into Intracranial Internal Carotid Arteries," Cerebrovascular Diseases, Vol. 24, No. 2-3, 2007, pp. 314-315. doi:10.1159/000106517

[4] I. Asouhidou and T. Asteri, "Acute Aortic Dissection: Be aware of Misdiagnosis," BMC Research Notes, Vol. 2, No. 2, 2009, p. 25. doi:10.1186/1756-0500-2-25

[5] A. L. Estrera, Z. Garami, C. C. Miller, E. E. Porat, P. E. Achouh, J. Dhareshwar, R. Meada, A. Azizzadeh and H. J. Safi, “Acute Type A Aortic Dissection Complicated by 
Stroke: Can Immediate Repair Be Performed Safely?” Journal of Thoracic and Cardiovascular Surgery, Vol. 132, No. 6, 2006, pp. 1404-1408. doi:10.1016/j.jtcvs.2006.07.026

[6] C. Gaul, W. Dietrich and F. J. Erbguth, "Neurological Symptoms in Aortic Dissection: A Challenge for Neurologists,” Cerebrovascular Diseases, Vol. 26, No. 1, 2008, pp. 1-8.

[7] M. Grupper, A. Eran and A. Shifrin, "Ischemic Stroke, Aortic Dissection, and Thrombolytic Therapy-The Importance of Basic Clinical Skills,” Journal of General Internal Medicine, Vol. 22, No. 9, 2007, pp. 1370-1372. doi:10.1007/s11606-007-0269-2

[8] T. Ngernsritrakul and P. Sathirapanya, "Type A Aortic Dissection Presenting as Acute Ischemic Stroke Caution for Thrombolytic Therapy: A Case Report and Literatures Review," Journal of The Medical Association of Thailand, Vol. 91, No. 8, 2008, pp. 1302-1307.

[9] S. Takeuchi, Y. Takasato, H. Masaoka and N. Otani, "Administration of Recombinant Tissue Plasminogen Activator to a Case of Cerebral Infarction in the Setting of Painless Aortic Dissection,” Neurology India, Vol. 57, No. 6, 2009, pp. 808-809. doi:10.4103/0028-3886.59487

[10] K. D. Flemming and R. D. Jr. Brown, “Acute Cerebral Infarction Caused by Aortic Dissection: Caution in the Thrombolytic Era,” Stroke, Vol. 30, No. 2, 1999, pp. 477-478. doi:10.1161/01.STR.30.2.477

[11] J. F. Yeh, H. Po and C. Y. Chien, "Ischemic Infarction
Masking Aortic Dissection: A Pitfall to Be Avoided before Thrombolysis,” Emergency Medicine Journal, Vol. 24, No. 8, 2007, pp. 594-595. doi:10.1136/emj.2007.046904

[12] Y. Iguchi, K. Kimura, K. Sakai, N. Matsumoto, J. Aoki, S. Yamashita and K. Shibazaki, "Hyper-Acute Stroke Patients Associated with Aortic Dissection,” Internal Medicine, Vol. 49, No. 6, 2010, pp. 543-547.

[13] J. Y. Kwon, J. H. Sung, I. S. Kim and B. C. Son, "Painless Dissecting Aneurysm of the Aorta Presenting as Simultaneous Cerebral and Spinal Cord Infarctions," Journal of Korean Neurosurgical Society, Vol. 50, No. 3, 2011, pp. 252-255. doi:10.3340/jkns.2011.50.3.252

[14] D. Rodríguez-Luna, R. M. Vilar, M. Peinazo, A. del Villar, B. Claramonte, C. Vilar and D. Geffner, "Intravenous Thrombolysis in an Elderly Patient with Acute Ischemic Stroke Masking Aortic Dissection,” Journal of Stroke and Cerebrovascular Diseases, Vol. 20, No. 6, 2011, pp. 559-561.

[15] O. O. Zaidat, E. E. Ubogu and A. J. Lerner, "Recurrent Transient Ischemic Attacks as the Initial Presenting Manifestation of Type A Aortic Dissection,” Annals of Vascular Surgery, Vol. 16, No. 5, 2002, pp. 676-678. doi:10.1007/s10016-001-0193-6

[16] V. Wright, R. Horvath and A. E. Baird, "Aortic Dissection Presenting as Acute Ischemic Stroke,” Neurology, Vol. 61, No. 4, 2003, pp. 581-582. 